\title{
How Can We Evaluate Disability without Bias?
}

\author{
Sunyoung Kim, Chang Won Won \\ Department of Family Medicine, Kyung Hee University Medical Center, Seoul, Korea
}

We read the article by Jang and Kawachi ${ }^{1)}$ regarding cultural and sexual differences in activities of daily living (ADLs) and instrumental activities of daily living (IADLs) with great interest. The authors concluded that cross-national comparisons of $\mathrm{ADL}$ and IADL disabilities need to consider item response bias stemming from culture- or education-based gender differences in household type and cognitive IADLs. While we agree that IADLs have gender- or culture-specific biases by nature, the results of this paper did not seem to demonstrate these biases in ADL items; thus, questions still remain for the conclusion.

We also agree with the author's suggestion that "unless corrected for, cross-national variations in disability rates may reflect item-response bias rather than real differences in disability levels". However, the last sentence "If possible, a culture-neutral ADL and IADL measurement that does not require DIF analyses should be developed" leaves room for consideration. Again, ADLs are not culture dependent, and IADL items should be based on each country's culture. ${ }^{2)}$ For example, the IADL items in the UK include whether one can brew tea and bring ashtrays, while those in New Zealand include gardening. ${ }^{3,4)}$ Therefore, national differences are inevitably a part of considering the cultural life of the residents and should be selected as an item that considers the characteristics of each country rather than simple translation-reverse translation in that country.

Meanwhile, Jung et al. ${ }^{5)}$ reported that men were more dependent on cooking and doing laundry, while women were more dependent on going out, using transportation, shopping, managing money, and using cell phones. However, this dependence was not significant in multiple regression analysis, and after adjusting for age, education, and comorbidities, IADLs showed a significantly lower dependence rate in women. ${ }^{5)}$ These results differed from those of previous studies ${ }^{6-10)}$ that reported many ADL functional disorders in older women. Indeed, Sheehan et al. ${ }^{11)}$ reported significant negative cohort slopes for men and women for not using a map and for men in preparing meals and shopping even after adjusting for age, ethnicity, education, and marital status. These results were consistent with those of a previous research that suggests that households are becoming more egalitarian and that by assuming increasing gender egalitarianism in household tasks, going forward, there should be less potential bias in IADL measures. ${ }^{12)}$

As proposed by Jang et al., ${ }^{1)}$ owing to gender bias, the assessment in some studies included only 5-6 items after excluding items not implemented in men. ${ }^{13)}$ As the initial IADL assessment by Lawton and Brody et al. comprised seven items (telephone, transportation, shopping, meal preparation, household chores, taking medicine, and money management), they did not investigate food preparation, housework, or laundry in men. ${ }^{14)}$

Finally, one important thing to consider is that disability (capability) of IADL and dependency of IADL are somewhat different. By definition, disability is any condition that makes it more "difficult" for a person to perform certain activities, which encompasses impairments, activity limitations, and participation restrictions. ${ }^{15)}$ In this paper, if the respondents were partly or totally dependent for a given activity, they were categorized as having IADL disability. However, we would propose that IADL dependency is more correct in these instances. A man can be dependent on others for cooking even though he actually can perform this task.

For example, when performing the K-ADL and K-IADL questionnaire, rather than asking "whether it is possible (ability)", the question should be "whether it is actually being done (implementation)" to determine how much help (care) is needed rather than potential capabilities. In particular, men will often not report "performing household chores", "preparing meal", "doing laundry", and "managing money" items based on social conventions or because others perform these tasks for them. To cope with these problems, those who answered "don't do" to these items were instructed to further ask "if they could" or "they had never done so" in the original K-IADL. 
Overall, we should carefully assess disability measures and utilize, clearly consider, and investigate how changes in the social expectations of engaging in the queried activities can affect outcomes. As society rapidly changes its individuals' roles, expectations, environments, and other characteristics, developing new measures without bias will become increasingly important to understand the health of aging populations.

\section{ACKNOWLEDGMENTS}

\section{CONFLICT OF INTEREST}

The authors claim no conflicts of interest.

\section{REFERENCES}

1. Jang SN, Kawachi I. Why do older Korean adults respond differently to activities of daily living and instrumental activities of daily living? A differential item functioning analysis. Ann Geriatr Med Res 2019;23:197-203.

2. Brena SF, Sanders SH, Motoyama H. American and Japanese chronic low back pain patients: cross-cultural similarities and differences. Clin J Pain 1990;6:118-24.

3. Fillenbaum GG. Screening the elderly: a brief instrumental activities of daily living measure. J Am Geriatr Soc 1985;33:698-706.

4. Katz S, Ford AB, Moskowitz RW, Jackson BA, Jaffe MW. Studies of illness in the aged. The index of ADL: a standardized measure of biological and psychosocial function. JAMA 1963;185:914-9.

5. Jung JY, Kim JS, Choi HJ, Lee KY, Park TJ. Factors associated with $\mathrm{ADL}$ and IADL from the third Korea National Health and Nutrition Examination Survey (KNHANES III), 2005. Korean J Fam Med 2009;30:598-609.

6. Ng TP, Niti M, Chiam PC, Kua EH. Prevalence and correlates of functional disability in multiethnic elderly Singaporeans. J Am Geriatr Soc 2006;54:21.-9.

7. Dorantes-Mendoza G, Avila-Funes JA, Mejia-Arango S, Gutierrez-Robledo LM. Factors associated with functional dependence in older adults: a secondary analysis of the National Study on
Health and Aging, Mexico, 2001. Rev Panam Salud Publica 2007;22:1-11.

8. Lee KJ, Park HS. A study on the perceived health status, depression, and activities of daily living for the elderly in urban areas. Korean J Women Health Nurs 2006;12:221-30.

9. Murtagh KN, Hubert HB. Gender differences in physical disability among an elderly cohort. Am J Public Health 2004;94:140611.

10. Leveille SG, Resnick HE, Balfour J. Gender differences in disability: evidence and underlying reasons. Aging (Milano) 2000; 12:106-12.

11. Sheehan C, Domingue BW, Crimmins E. Cohort trends in the gender distribution of household tasks in the United States and the implications for understanding disability. J Aging Health 2019;31:1748-69.

12. Artis JE, Pavalko EK. Explaining the decline in women's household labor: Individual change and cohort differences. J Marriage Fam 2003;65:746-61.

13. Jekel K, Damian M, Wattmo C, Hausner L, Bullock R, Connelly PJ, et al. Mild cognitive impairment and deficits in instrumental activities of daily living: a systematic review. Alzheimers Res Ther 2015;7:17.

14. Lawton MP, Brody EM. Assessment of older people: self-maintaining and instrumental activities of daily living. Gerontologist 1969;9:179-86.

15. World Health Organization. Disability [Internet]. Geneva, Switzerland: World Health Organization; c2020 [cited 2020 Jun 10]. Available from: https://www.who.int/health-topics/disability\#tab=tab_1.

Corresponding Author: Chang Won Won, $\mathrm{MD}, \mathrm{PhD}$

Department of Family Medicine, Kyung Hee University Medical Center, 23

Kyungheedae-ro, Dongdaemun-gu, Seoul 02447, Korea

E-mail: chunwon@khmc.or.kr

ORCID: https://orcid.org/0000-0002-6429-4461

Received: May 21, 2020; Accepted: June 2, 2020 
